\title{
Switching between memory and perception: Moving attention or memory retrieval?
}

\author{
VERONICA J. DARK \\ Iowa State University, Ames, Iowa
}

\begin{abstract}
Weber, Burt, and Noll (1986) estimated that the time needed to switch attention between memory and perception was around $300 \mathrm{msec}$. The first two experiments in the present paper estimated switching time using a variation of their task. Subjects reported aloud lists of six items. The items were read off a computer screen (perception), recited from memory, or reported alternately from the two sources. The data show that the switching-time estimate is influenced by input/output compatibility, response-initiation times, and memory load. When these factors were controlled, estimated switching time dropped to around 100-150 msec. The data suggest, how ever, that the switch from perception to memory might be slower than the switch from memory to perception, which would invalidate the formula used to compute switching time. Experiment 3 tested the time for a single switch from perception to memory and a single switch from memory to perception by restricting report to one pair of items in the list. When the to-be-reported pair was precued, estimated switching time dropped to zero. When the pair was not precued, the memory-to-perception switching time remained at zero, but the perception-to-memory time was more than $400 \mathrm{msec}$. The pattern of results forced a reconceptualization of the task in terms of memory retrieval rather than attention switching. The attention-switching times appear to reflect processes required to select items from memory.
\end{abstract}

Weber, Burt, and Noll (1986) pointed out that many tasks require rapid switching of attention between perception and memory; that is, the tasks are structured so that information in the environment and information in memory must be alternately accessed. Using the workingmemory conceptualization of Baddeley (1982; Baddeley \& Hitch, 1974), Weber et al. described a model in which attention switching is the passing of control between memory and perception subroutines. Attention was figuratively described as a switch that could be moved between perceptual information in a visual/spatial scratchpad and memorial information in an articulatory/phonetic scratchpad. Items to which the switch was directed could be accessed by the control processes of working memory and output as responses. When a task required that perceptual and memorial information be intermixed at output, the switch would have to alternate rapidly between the two sources. Weber et al. were interested in the time necessary to accomplish the switch between memory and perception and whether switching required any of the limitedcapacity control processes available to working memory.

This research was partially funded by DHHS/NIH Grant 2 S07 RR07034-21 to the Iowa State University Office of the Vice President for Research. I would like to thank Pamela Atkinson, Jennifer Grant, Clay Helberg, and Susan Miguel for collecting the data reported in this paper. Also, thanks to William Johnston, Lloyd Avant, Robert Weber, and two anonymous reviewers for helpful comments. Portions of these data were presented at the Meeting of the Midwestern Psychological Association in Chicago, May 1989. Correspondence concerning this article may be addressed to Veronica J. Dark, Department of Psychology, Iowa State University, Ames, IA 50011.
Weber et al. (1986, Experiment 1) estimated switching time by comparing oral production times for lists of letters three or six items long. All-memory lists of either three or six items were recited aloud after they were silently studied. All-perception lists of either three or six items were read off a computer screen. Mixed lists consisted of a three-item memory sublist and a three-item perception sublist. The subject alternated report of items from the two sublists, thus switching between memory and perception five times in producing the full six-item list.

Weber et al. (1986) computed the average switching time by two formulas. With the first formula, they computed the difference between average production latency for the six-item mixed lists and the summed production latencies for the two unmixed three-item lists. With the second formula, they computed the difference between the six-item mixed lists and the average of the two unmixed six-item lists. In each case, the difference was divided by five in order to determine the mean time per switch.

The estimated switching times from the two formulas were 293 and $377 \mathrm{msec}$, respectively. Weber et al. (1986) attributed the difference to faster per-item production times with the unmixed six-item versus three-item lists. They concluded that the first formula was more conservative as well as more flexible, and used it in a second experiment in which they examined the impact of list length on switching times. They found that estimated switching time increased with increases in list length. Weber et al. interpreted the increase as indicating that both moving the switch and maintaining items in memory required limited-capacity working-memory resources. 
In both formulas used by Weber et al. (1986), the production times for unmixed lists, which reflect the time to move attention between items within memory or within perception, are subtracted from the production times for mixed lists, which reflect the additional time needed to move attention between the two modalities (or scratchpads). Both formulas produced large, positive switching times. The data, therefore, were interpreted as showing that items in one modality are in some way closer to each other than to items in the other modality, and that the distance between items affects the time needed to move attention.

Examination of Weber et al.'s (1986) procedure, however, reveals some problems that may have inflated the estimated switching times. One problem is a difference in input/output compatibility between the mixed and unmixed lists. Weber et al. presented the items in a single row on a computer screen. The output order for the unmixed lists was the same order as input; that is, the subject read the items or recited them from memory in the order they were presented on the screen. Items from mixed lists, however, were output in a different order than they were input. The mixed lists essentially consisted of two separate sublists-a three-item memory list and a threeitem perception list-thus, alternation between memory and perception was confounded with keeping track of position within two lists rather than just one. The increased task difficulty in the mixed lists resulting from the confound may have led to an increase in production latency and an increase in the estimated switching time. In Experiment 1 of the present paper, that confound was removed.

\section{EXPERIMENT 1}

Experiment 1 required between-row alternation in both the unmixed and the mixed conditions. The six items comprising each list were presented in two rows, or sublists, of three items each. The subject was required to say aloud the items from left to right while alternating between the top and bottom rows. On all-memory trials, each item was recited from memory. On all-perception trials, each item was read from the computer screen. On mixed trials, items in the top row were read from the computer screen, and items in the bottom row were recited from memory.

\section{Method}

Subjects. Twenty students at Iowa State University received extra credit in an undergraduate psychology class for participating as subjects. All subjects reported normal or corrected-to-normal vision.

Stimuli. On each trial, the subject reported aloud six letters. The letters were recited from memory, read off the screen, or both. Each trial began with the $250-\mathrm{msec}$ presentation of a ready signal (two "plus" signs) in the center of a computer screen. The ready signal was followed, after a 150 -msec blank interval, by the first stimulus display. Display 1 provided the memory items, if any, and remained on for $4 \mathrm{sec}$. It was followed, after a 150 -msec blank interval, by Display 2. Display 2 provided the perception items, if any, and constituted the signal to begin responding. The message "RESPOND NOW" occurred as a reminder under the bottom stimulus row of Display 2. As soon as the subject finished saying the last letter, he or she pressed the space bar on the computer keyboard. Production latency for the list was the time between onset of Display 2 and the barpress. The intertrial interval was $2 \mathrm{sec}$. The timing within a trial and the use of a barpress to signal that the list had been produced were similar to the procedures of Weber et al. (1986).

Each stimulus display consisted of two rows of three characters. The characters were either asterisks or to-be-reported items. There were blank spaces between the characters on each row and there was a blank line between the two rows. Display 1 appeared centered on the screen. The characters of Display 2 appeared in the same locations as the corresponding characters in Display 1 . The three types of list differed in terms of which display(s) contained the to-be-reported items. Asterisks were presented in each position of the display that did not contain a to-be-reported item.

In all-memory lists, the six items were presented in Display 1. In all-perception lists, the six items were presented in Display 2. In the mixed lists, the items comprising the bottom row were presented in Display 1 and the items comprising the top row were presented in Display 2. Thus, the mixed lists can be described as perception-first/five-switch lists: the response began with a perception item and alternation between the rows required five switches between memory and perception. Examples of both displays for the three types of list are shown in the top half of Table 1.

Procedure. The subjects read instructions that described and then illustrated the three types of lists. The instructions indicated that the appropriate response sequence was to alternate between rows while going from left to right, and emphasized both speed and accuracy. A practice set of six lists contained two lists of each type.

Stimulus lists were constructed from the pool of 10 nonconsecutive consonants (B, D, F, H, J, L, N, P, R, and T) used by Weber et al. (1986). Each stimulus list consisted of 6 of the 10 letters selected randomly and without replacement. Ninety-six lists were generated (in addition to the six practice lists) and grouped into blocks of six. Each block contained two all-memory lists, two all-perception lists, and two mixed (perception-first/five-switch) lists. The order of the six lists within each block was individually randomized for each subject. The first block of lists was discarded as additional practice, leaving 30 experimental lists of each type.

Randomization, stimulus presentation, timing, and recording of production latencies was accomplished via the APT II software package (Foltz \& Poltrock, 1985; Poltrock \& Foltz, 1982) implemented

Table 1

Examples of Display 1 and Display 2 for Each Type of List in Experiments 1, 2, and 3

\begin{tabular}{ccc}
\hline Type of List & Display 1 & Display 2 \\
\hline All perception & $* * *$ & R N F \\
All memory & $* * *$ & HG L \\
& $\mathrm{R} \mathrm{NF}$ & $* * *$ \\
Perception first/ & H G L & $* * *$ \\
five switch & H G & R N F \\
Memory first/ & $\mathrm{R} \mathrm{N} \mathrm{F}$ & $* * *$ \\
five switch & $* * *$ & $* * *$ \\
Perception first/ & $* * \mathrm{~F}$ & $\mathrm{HG} \mathrm{L}$ \\
one switch & $* \mathrm{GL}$ & $\mathrm{R} \mathrm{N} *$ \\
Memory first/ & $\mathrm{R} \mathrm{N} *$ & $\mathrm{H} * *$ \\
one switch & $\mathrm{H} * *$ & $* * \mathrm{~F}$ \\
\hline
\end{tabular}

Note-The correct response in all cases is RHNGFL. The phrase "RESPOND NOW" also occurred under the second row as part of Display 2 in all lists. Only the first three list types occurred in Experiment 1, all six occurred in Experiment 2, and the first four occurred in Experiment 3. 
on an Apple Ile computer equipped with a Timemaster II clockcard and a Zenith (ZVM121) monitor. Response accuracy was scored by the experimenter.

The entire procedure, including debriefing, took less than $30 \mathrm{~min}$.

\section{Results and Discussion}

Unless otherwise stated, $p<.05$ for all significant differences and $p>.10$ for all nonsignificant differences for all analyses in all experiments. Note also that the latency data include only trials on which the correct response was produced. ${ }^{1}$

Response accuracy and production latency for the three types of list are shown in Table 2 . The list types differed significantly on accuracy $\left[F(2,38)=38.60, M S_{\mathrm{e}}=.010\right]$ and on latency $\left[F(2,38)=55.95, M S_{e}=44,332\right]$. Neuman-Keuls comparisons among the three means for each measure showed that each mean differed from the other two.

Switching time was computed via the second formula of Weber et al. (1986), the only formula possible with the equal-length lists required by the present design. That is, the average of the two unmixed conditions was subtracted from the mixed condition, and the result was divided by five because there were five switches between memory and perception in each mixed list. The obtained value of $155 \mathrm{msec}$ was much shorter than the equivalent value of $377 \mathrm{msec}$ reported by Weber et al. Because the requirement to keep track of ordinal position in two sublists is not confounded with alternating between perception and memory in the present experiment, as it was in the Weber et al. study, the obtained value is a better estimate of the additional time needed to move attention between memory and perception. Although there is no necessity for a match, the value is also much closer to those in the spatial attention literature in which attention is switched between locations in response to a precue (e.g., Eriksen \& St. James, 1986; Murphy \& Eriksen, 1987).

As might be expected, the subjects were less accurate in reciting six items from memory than in reading six items off the screen, and performance in the mixed condition was in between. In contrast, reciting six items from memory took less time than did reading six items. The same pattern was present in the data of Weber et al. (1986); that is, all-memory lists always showed the fastest production latencies (although the difference was not always reliable).

Table 2

Mean Accuracy (Proportion Correct), Production Latency (in msec), and Latency Standard Deviations for the Three Types of Lists in Experiment 1

\begin{tabular}{|c|c|c|c|c|c|c|}
\hline \multirow[b]{2}{*}{ Type of List } & \multicolumn{2}{|c|}{ Accuracy } & \multicolumn{2}{|c|}{$\begin{array}{l}\text { Production } \\
\text { Latency }\end{array}$} & \multicolumn{2}{|c|}{$\begin{array}{l}\text { Standard } \\
\text { Deviation }\end{array}$} \\
\hline & $M$ & $S D$ & $M$ & $S D$ & $M$ & $S D$ \\
\hline All perception & .969 & .039 & 2,723 & 626 & 434 & 156 \\
\hline All memory & .723 & .140 & 2,390 & 643 & 564 & 232 \\
\hline Mixed & .855 & .117 & 3,068 & 630 & 679 & 160 \\
\hline
\end{tabular}

Note-Mixed refers to a perception-first/five-switch list.
The phenomenon could be the result of a speed/accuracy tradeoff in which the lists missed in the all-memory condition are primarily lists that take longer to produce. In addition to a faster overall average latency, a speed/accuracy tradeoff should result in a less variable range of responses for each subject over the all-memory lists. This possibility was tested by computing for each subject the standard deviation associated with the correct latencies for each type of list. The average value of those standard deviations is reported in Table 2. Although there was a significant difference among the types of list $[F(2,38)=12.52$, $\left.M S_{e}=24,065\right]$, in comparison to the all-perception lists, there was more variability, rather than less, with the allmemory lists.

Another possibility for the faster production of allmemory lists is that information in memory is more quickly available to the response system than is information in the environment. The pattern, however, is more likely the result of the task requirements. With all-memory lists, the subjects had all information regarding the exact response sequence before they were given the cue to produce the list. To the extent that letter-specific response processing must precede the initiation of the vocal response, the all-memory lists would benefit. The preprocessing could be carried out before the cue to produce the list was presented, and the subjects could then more quickly initiate the response. The potential contribution of this factor is not taken into account when switching time is estimated. If all-memory lists are artificially fast, the unmixed latency average will be artificially fast and the switching time will be overestimated. The hypothesis that the difference in production latency between the all-perception and the allmemory lists is due to response initiation rather than response production is examined as part of Experiment 2.

\section{EXPERIMENT 2}

Experiment 1, in conjunction with the data of Weber et al. (1986), revealed the sensitivity of the list-production task to a mismatch between input order and output order. When the input/output relationship was equated for both mixed and unmixed lists, the estimated switching time dropped dramatically. Although the lists were equated on this factor in Experiment 1, another potential problem is differences in memory load.

The formulas of Weber et al. (1986) assume that the effects of memory load on production time are additive. In the currently used formula, for example, performance on six-item all-perception lists with zero memory load is averaged with performance on six-item all-memory lists and compared to performance on six-item mixed lists in which the memory load is three. Estimated switching times increase with list length (Weber et al., 1986, Experiment 2), but memory load also increases with list length. Thus, the possibility exists that the additivity assumption may not be appropriate. A more nearly ideal situation would equate memory load on mixed and unmixed lists. 
Six types of list were used in Experiment 2 in order to examine the impact of memory load on estimated switching time. As in Experiment 1, there were allmemory, all-perception, and perception-first/five-switch lists. Three additional types of load-three mixed lists were also included: (1) memory-first/five-switch lists, (2) perception-first/one-switch lists, and (3) memoryfirst/one-switch lists. The one-switch lists were constructed so that the response for each list began with either three memory items or three perception items and ended with three of the other type of item. Examples of the three additional list types are shown in the bottom half of Table 1. Note that the switching time can be estimated entirely from trials with the same memory load by taking the difference between the one-switch and the five-switch conditions and dividing it by four.

Two latencies were recorded for each trial in Experiment 2: an initiation latency and an execution latency. The initiation latencies provided data relevant to the hypothesis that preprocessing of responses on trials beginning with memory items produces quicker overall latencies because of quicker response initiation. The logic upon which the switching-time formula is based requires that the latencies entered into the formula reflect only response-production time. To the extent that the latencies for memory lists in Experiment 1 were shorter because of quicker response initiation, switching time would be overestimated. Execution latencies, uncontaminated by differences in response initiation, were used to estimate switching times in Experiment 2 .

\section{Method}

Subjects. Twenty students at Iowa State University received extra credit in an undergraduate psychology class for participating as subjects. All subjects reported normal or corrected-to-normal vision. No subject had been in Experiment 1.

Stimuli. The equipment and the basic procedure were as described for Experiment 1. The only difference in timing between experiments resulted from measuring response-initiation latency. A voice key hooked into the paddle input on the Apple Ile computer provided the input used to define initiation latency. The APT II software required a brief blank period of $150 \mathrm{msec}$ during which this latency was recorded. As a result, the presentation of Display 2 was interrupted by a $150-\mathrm{msec}$ blank interval that the subjects saw as a brief flicker. As soon as the subjects finished saying the list, they pressed the space bar. Barpress latency, timed from the resumption of Display 2 after the 150 -msec interruption, was the measure of responseexecution time.
Procedure. The instructions contained six examples, one of each type of list. The subjects were told to observe Display 1 and remember any memory letters, to say aloud all six letters in order from left to right while alternating from top to bottom as soon as Display 2 appeared, and to press the space bar as soon as they had finished responding. The instructions described the two latency measures and informed the subject about the flicker, but they also stressed accuracy. There were six practice lists, one of each type, before the actual experiment began.

Ninety lists were generated as in Experiment 1 and were grouped into 15 blocks of six. Each block contained one of each type of list. The order of lists within each block was randomized individually for each subject. The first block was discarded as additional practice, leaving 14 lists in each condition.

The entire session, including debriefing, took less than $30 \mathrm{~min}$.

\section{Results and Discussion}

Accuracy, response-initiation latency, and responseexecution latency for the six types of list are presented in Table 3. Each measure will be discussed separately. Note that total production time, comparable to that reported in Experiment 1, would equal the initiation latency plus the execution latency plus $150 \mathrm{msec}$.

Accuracy. An analysis of variance (ANOVA) of the accuracy data with type of initial item (memory vs. perception) and number of switches (zero, one, or five) as within-subject factors showed an effect of initial item $\left[F(1,19)=6.77, M S_{\mathrm{e}}=.011\right]$, an effect of number of switches $\left[F(2,38)=9.56, M S_{\mathrm{e}}=.018\right]$, and an interaction between these factors $\left[F(2,38)=39.71, M S_{e}=.012\right]$. A Newman-Keuls comparison among the means showed that (1) accuracy was highest for the all-perception lists and the memory-first/one-switch lists, (2) there was no significant difference in accuracy between the perceptionfirst/one-switch lists and both types of five-switch lists, and (3) accuracy in the all-memory list was significantly lower than accuracy in the perception-first/one-switch list but only marginally lower than in the two five-switch lists $(.05<p<.10)$

It is not surprising that the all-memory lists produced the lowest accuracy, nor that the all-perception lists produced high accuracy. Somewhat unexpected, however, was that accuracy in the memory-first/one-switch lists was just as high as in the all-perception lists. The other lists with a memory load of three produced an intermediate level of accuracy, as expected. The accuracy pattern among the load-three lists suggests the operation of out-

Table 3

Mean Accuracy (Proportion Correct), Initiation Latency (in msec), and Execution Latency (in msec) for the Six Types of Lists in Experiment 2

\begin{tabular}{|c|c|c|c|c|c|c|}
\hline \multirow[b]{2}{*}{ Type of List } & \multicolumn{2}{|c|}{ Accuracy } & \multicolumn{2}{|c|}{$\begin{array}{l}\text { Initiation } \\
\text { Latency }\end{array}$} & \multicolumn{2}{|c|}{$\begin{array}{c}\text { Execution } \\
\text { Latency }\end{array}$} \\
\hline & $M$ & $S D$ & $M$ & $S D$ & $M$ & $\overline{S D}$ \\
\hline All perception & .960 & .046 & 1,010 & 305 & 1,389 & 213 \\
\hline All memory & .676 & .187 & 660 & 186 & 1,635 & 300 \\
\hline Perception first/one switch & .811 & .161 & 882 & 142 & 1,620 & 315 \\
\hline Memory first/one switch & .955 & .055 & 648 & 127 & 1,397 & 214 \\
\hline Perception first/five switch & .758 & .153 & 1,030 & 204 & 1,963 & 315 \\
\hline Memory first/five switch & .747 & .165 & 844 & 171 & 1,903 & 272 \\
\hline
\end{tabular}


put interference (e.g., Roediger, 1973, 1974; Roediger \& Schmidt, 1980); the difficulty arises when memory items must be output after several other items are output.

Initiation latency. An ANOVA of the initiation latencies, with type of initial item and number of switches as within-subject factors, showed an effect of initial item $\left[F(1,19)=117.37, M S_{\mathrm{e}}=16,815\right]$, an effect of number of switches $\left[F(2,38)=18.46, M S_{\mathrm{e}}=16,209\right]$, and an interaction between these factors $[F(2,38)=6.90$, $\left.M S_{\mathrm{c}}=10,383\right]$. A Newman-Keuls comparison among the means showed that (1) the fastest initiation latencies occurred with the all-memory lists and the memory-first/oneswitch lists, (2) intermediate latencies occurred with the memory-first/five switch lists and the perception-first/oneswitch lists, and (3) the slowest latencies occurred with the all-perception and the perception-first/five-switch lists.

Responses that began with memory items were initiated more quickly than responses beginning with perception items. The benefit was especially large when the response began with three or six memory items rather than one, as would be expected from the hypothesis that preprocessing of specific response sequences is possible on memory-first trials. No list-initiation differences among the perceptionfirst trials were expected as a function of the number of switches. Initiation latency for the perception-first/oneswitch lists, however, was faster than for the other two types of lists beginning with perception items.

The initiation-latency differences support the hypothesis that preprocessing specific responses during Display 1 contributed to the faster total production latency for the all-memory lists in Experiment 1. Response-initiation differences from preprocessing specific responses are not taken into account in the attention-switching formulas of Weber et al. (1986). The benefit appears to increase with the number of items that can be preprocessed.

Execution latency. Execution latency was the time between the initiation of the response and its completion. It should reflect both the time necessary to say the final five letters of the list and the time necessary for zero, one, or five switches, depending on list type. An ANOVA with initial item and number of switches as within-subject factors showed an effect of number of switches $[F(2,38)=$ $\left.40.74, M S_{e}=58,471\right]$ and an interaction between number of switches and type of initial item $[F(2,38)=23.59$, $\left.M S_{\mathrm{e}}=24,047\right]$. There was no main effect of type of initial item $(F<1)$. A Newman-Keuls comparison among the means showed that (1) the fastest latencies occurred in the all-perception lists and the memory-first/one-switch lists, (2) intermediate latencies occurred in the all-memory lists and the perception-first/one-switch lists, and (3) the slowest latencies occurred in the two five-switch lists.

The design of Experiment 2 allowed several different ways of estimating attention-switching time. An estimate derived solely from the lists with a memory load of three was computed by subtracting the one-switch latency from the five-switch latency (both averaged over type of initial item) and dividing by four (because the lists differ by four switches). The switching-time estimate was $106 \mathrm{msec}$; it was marginally longer than the 84-msec estimate computed from the difference in latency between five-switch lists and unmixed lists $\left[t(19)=2.07, S_{\mathrm{e}}=10.6, .05<p<\right.$ .10]. Thus, there is some support for the assertion that the switching-time formula is sensitive to memory load. Combining unmixed lists with loads of zero and six so that they can be compared to mixed lists with a load of three may lead to a slight underestimation of switching time.

The switching-time estimates derived by averaging over type of initial item give a misleading picture of regularity not found in the latencies. With both the one-switch and the unmixed lists, the two averaged latencies are unequal. The picture becomes complex, however, if switching times are computed separately. Switching time for perception-first trials can be estimated by taking the difference between the perception-first/five-switch lists and the perception-first/one-switch lists and dividing by four. The switching-time estimate of $86 \mathrm{msec}$ is significantly shorter than the estimate of $126 \mathrm{msec}$ derived via the same formula from memory-first trials $\left[t(19)=3.04, S_{\mathrm{e}}=13.5\right]$.

Note that one can get a more direct measure of switching time (but one derived from lists with different memory loads) by taking the difference between the unmixed and the one-switch lists. The switching time of $-7 \mathrm{msec}$ obtained by averaging over type of initial item suggests that it does not take more time to move attention between memory and perception than to move it among items in either modality. The picture changes again when switching time is computed without averaging. The difference between the memory-first/one-switch lists and the allmemory lists should reflect the time needed to move attention from memory to perception. The difference was $-238 \mathrm{msec}$; that is, lists with a switch were produced more quickly than lists without a switch. The difference between perception-first/one-switch lists and all-perception lists should reflect the time needed to move attention from perception to memory. The difference was $231 \mathrm{msec}$; that is, lists with a switch took longer than lists without a switch.

Type of initial item does not appear to matter for the five-switch lists, but a one-switch list beginning with three memory items is more like an all-perception list. Likewise, a one-switch list beginning with three perception items is more like an all-memory list. Interpreted within the context of the model of Weber et al. (1986), the data suggest that switching from memory to perception may not be the same as switching from perception to memory. If that were true, it would invalidate the logic upon which the formula for estimating switching time is based. It is also possible that something other than attention switching is responsible for the pattern of latencies. An alternative will be described in the General Discussion.

The results of Experiments 1 and 2 confirm that the estimates of attention-switching time produced by Weber et al. (1986) were overly long. Their estimates appear to have included latency differences attributable to input/output compatibility, memory load, and response initiation. 
When these factors are more equivalent across conditions, as in the present Experiments 1 and 2, estimated switching times are much lower.

The fact that such factors can so easily affect the estimated switching times, however, is problematic. The lists must be carefully equated if the estimated switching time is to reflect only the time to move attention between modalities. An additional problem is that the switchingtime formula assumes that a memory-to-perception switch is equal to a perception-to-memory switch. The data of Experiment 2 suggest that this assumption is wrong. Direct measurement of the time to make a single memoryto-perception switch and a single perception-to-memory switch would provide a straightforward test of the assumption. Measuring a single switch might also lessen the influence of other factors on the obtained switching times. The time to make a single switch in each of the two directions was measured in Experiment 3.

\section{EXPERIMENT 3}

Weber et al. (1986) suggested that tasks in which there is only one switch of attention between memory and perception might not accurately reflect the switching that occurs in continuous tasks. They argued that continuous alternation tasks might require loading and flushing of buffers and that this might affect the time to switch attention. Although the point is well taken, information obtained from single-switch conditions could be useful in distinguishing between the contributions of the two potentially separate aspects of attention: attention as the mechanism that accesses a modality (i.e., attention as passing control to a subroutine) versus attention as the mechanism that determines which item, among those available in the modality, should be selected.

Stated in terms of the Weber et al. (1986) model, in mixed lists, attention must be directed toward the appropriate scratchpad (modality), and then the appropriate item must be selected from the scratchpad. In unmixed lists, only the latter step is necessary. The difference underlies the formulas for estimating switching time. Thus, moving attention can be conceptualized as a twostage process between modalities and a one-stage process within modalities. A perception-to-memory switch could be more difficult than a memory-to-perception switch in either or both steps.

In Experiment 3, subjects were presented with six-item lists similar to those in Experiment 1, but only one pair of letters was reported. The to-be-reported letter pair was indicated via carat markers appearing above and below the pair. A pair was either an unmixed perception/ perception or memory/memory pair, or a mixed perception/ memory or memory/perception pair.

Two groups of subjects differed in the timing of the markers. For the precue group, the markers came on $750 \mathrm{msec}$ before Display 2 and remained on during Display 2 . For the simultaneous cue group, the markers occurred only as part of Display 2 . The timing difference allowed partial separation of the impact of pair type on the two components of attention.

The precue subjects, like the subjects in Experiments 1 and 2, were able to select the appropriate modality before Display 2, and, when a memory item was first, they were able to preprocess the actual item to be output first. In addition, on mixed lists, they were able to selectively maintain in memory only the to-be-reported memory item regardless of whether it would be output first or second. In essence, the precue allowed the memory load to be reduced to one in such lists so that there should be no selection problem once memory was accessed, even in the perception-to-memory switching condition. Any difference between the unmixed and mixed pairs would, therefore, be attributable to moving attention between the two modalities as opposed to selection within a modality.

The simultaneous cue subjects, in contrast, could only move attention to the correct modality prior to Display 2 . For all pairs, they had to wait until Display 2 to select the appropriate items for output. The simultaneous cue subjects were thus effectively prevented from preprocessing specific responses involving memory items. As a result, production latency for pairs beginning with memory items was not expected to be faster than that for perception-first items. Differences between mixed and unmixed pairs for the simultaneous cue group could reflect both switching between modalities and selection within a modality. An effect found with the simultaneous cue group but not with the precue group, therefore, would implicate selection rather than attention switching.

\section{Method}

Subjects. Forty students at Iowa State University received extra credit for participating as subjects. All subjects reported normal or corrected-to-normal vision. No subject had participated in Experiments 1 or 2 . Twenty students were assigned to the precue group and 20 to the simultaneous cue group.

Stimuli. The subjects read instructions describing the task and illustrating examples of the four types of lists. The list types were the first four types described in Table 1: all-perception, all-memory, perception-first/five-switch, and memory-first/five-switch. The first item in each pair was from the top row and the second item was the corresponding letter in the bottom row. Thus, the unmixed pair types were either perception/perception or memory/memory, and the mixed pair types were either perception/memory or memory/ perception. The instructions emphasized both speed and accuracy. There were four practice lists, one of each type.

Procedure. The equipment and basic procedure were the same as in Experiment 1. For the simultaneous cue subjects, the timing was also the same: ready signal ( $250 \mathrm{msec}+150 \mathrm{msec}$ blank), Display 1 ( $4 \mathrm{sec}+150 \mathrm{msec}$ blank), and Display 2 (until the subject's keypress). For the precue subjects, however, Display 1 was presented for 3,250 msec (plus a 150-msec blank) and was followed by the precue display, which consisted only of the markers in the locations in which they would occur in Display 2. Display 2 occurred $750 \mathrm{msec}$ after the onset of the precue display. Thus, the total interval between onset of Display 1 and Display 2 was $4,150 \mathrm{msec}$ for both groups. Response-production time, from the onset of Display 2, was the only recorded latency. (Data from a pilot subject suggested that the software was unable to consistently measure both initiation and execution latency with the shorter response times obtained when just two items were reported.) 
Eighty-four lists were generated as in Experiment 1 and were grouped into 21 blocks of four lists each. One list within each block represented each type of list. The to-be-reported pair was chosen so that each position (left, right, and middle) was selected in at least one list per block, and over each set of three blocks, each position was assigned once to each type of list. The order of lists within each block was individually randomized for each subject. The first block was discarded as additional practice, leaving 20 lists in each condition.

The entire session, including debriefing, took less than $30 \mathrm{~min}$.

\section{Results and Discussion}

The accuracy and production latency for each type of pair for each group are presented in Table 4.

Accuracy was generally higher than in Experiments 1 and 2 , as might be expected because only two items were reported, but the pattern was similar. An analysis of the accuracy data, with group (precue vs. simultaneous cue) as a between-subjects factor and type of initial letter (memory vs. perception) and type of pair (unmixed vs. mixed) as within-subject factors, showed an effect of initial letter $\left[F(1,38)=39.54, M S_{e}=.004\right]$, an effect of type of pair $\left[F(1,38)=17.56, M S_{c}=.004\right]$, and an interaction between initial letter and type of pair $[F(1,38)=$ $\left.50.09, M S_{\mathrm{e}}=.007\right]$. There was no main effect of group, nor did group interact with the other factors. A NewmanKeuls comparison among the means showed that, for both groups, (1) accuracy was lowest with the memory/memory pairs, (2) accuracy with the perception/memory pairs was lower than with the perception/perception pairs, and (3) accuracy with the memory/perception pairs did not differ from either the perception/memory pairs or the perception/perception pairs.

A similar analysis of the latency data showed a very different pattern in which group interacted with all other effects. To simplify presentation, therefore, separate analyses are reported for each group.

Precue group. The only factor affecting latency for the precue group was the type of initial item $[F(1,19)=6.78$, $\left.M S_{e}=20,310\right]$. A Newman-Keuls comparison confirmed that the only difference was between the memory-first and the perception-first pairs. As would be expected from the preprocessing hypothesis, the subjects were faster when the initial item was reported from memory. The precue on memory-first pairs allowed the subject to do response preprocessing for at least one item of the pair. On perception-first pairs, the precue indicated only the spatial location of the first item, but it did allow selective storage of just one memory item with the perception/ memory pairs.

There was no latency difference between mixed and unmixed pairs. A difference would be expected if it takes more time to move attention between modalities than between items within a modality. The computed switching times were $-1 \mathrm{msec}$ for perception-first pairs and $-45 \mathrm{msec}$ for memory-first pairs. Neither was reliably different from zero (both $t \mathrm{~s}<1$ ). When item selection is simplified, moving attention between modalities does not appear to take any more time than does moving attention within modalities.

Simultaneous cue group. There was no reliable effect of initial item $\left[F(1,19)=2.31, M S_{e}=33,406\right]$, but there was an effect of type of pair $[F(1,19)=18.72]$ and an inital item $\times$ type of pair interaction $[F(1,19)=27.08$, $M S_{\mathrm{e}}=41,403$ for both]. A Newman-Keuls comparison showed that (1) the perception/perception pairs were produced most quickly, (2) the memory/memory pairs and the memory/perception pairs were produced at an intermediate speed, and (3) the perception/memory pairs were produced most slowly. Under conditions in which there was no advance information concerning the appropriate pair, the subjects were faster at selecting and reading two items off the screen than they were at selecting and reciting two items from memory. This phenomenon is not surprising because the cue is physically part of the perceptual display, and such cues have a powerful influence on attention (e.g., see Jonides, 1981).

As with the precue group, there was essentially no difference in reporting two items from memory in comparison with reporting one item from memory and then switching to perception; the switching time of $-31 \mathrm{msec}$ was not reliably different from zero $\left[t(19)=.37, S_{\mathrm{e}}=83.5\right]$. In contrast, there was a sizable difference $(443 \mathrm{msec})$ when the switch was from perception to memory. Because the precue group showed no perception-to-memory decrement, it appears that the decrement results from item selection and not from attention switching between modalities.

Table 4

Mean Accuracy (Proportion Correct), and Production Latency (in msec) for the Four Types of Pairs for Each Group in Experiment 3

\begin{tabular}{|c|c|c|c|c|c|c|c|c|}
\hline \multirow[b]{3}{*}{ Type of Pair } & \multicolumn{4}{|c|}{ Simultaneous Cue Group } & \multicolumn{4}{|c|}{ Precue Group } \\
\hline & \multicolumn{2}{|c|}{ Accuracy } & \multicolumn{2}{|c|}{$\begin{array}{c}\text { Production } \\
\text { Latency }\end{array}$} & \multicolumn{2}{|c|}{ Accuracy } & \multicolumn{2}{|c|}{$\begin{array}{c}\text { Production } \\
\text { Latency }\end{array}$} \\
\hline & $M$ & $S D$ & $M$ & $S D$ & $M$ & $S D$ & $M$ & $S D$ \\
\hline \multicolumn{9}{|l|}{ Unmixed } \\
\hline Perception/perception & 1.000 & .000 & 1,290 & 222 & .998 & .011 & 1,352 & 388 \\
\hline Memory/memory & .834 & .138 & 1,589 & 363 & .848 & .108 & 1,291 & 478 \\
\hline \multicolumn{9}{|l|}{ Mixed } \\
\hline Perception/memory & .933 & .109 & 1,733 & 265 & .957 & .067 & 1,350 & 346 \\
\hline Memory/perception & .974 & .035 & 1,558 & 229 & .982 & .038 & 1,245 & 367 \\
\hline
\end{tabular}


The data suggest that the attention-switching phenomena of Experiments 1 and 2 and Weber et al. (1986) are better conceptualized as within-memory selection phenomena.

\section{GENERAL DISCUSSION}

Weber et al. (1986) suggested that switching attention between perceptual information and memorial information could be described in terms of passing control between memory and perception subroutines. They attempted to measure the time it takes to make the switch. Experiments 1 and 2 were carried out within the context of their model. The results suggested that Weber et al. had overestimated the time necessary to switch attention between perception and memory in six-letter lists, and that the time was closer to $100-150 \mathrm{msec}$ than to the $300+\mathrm{msec}$ that they reported.

The data also suggested that switching time might depend on the direction of the switch, with the switch from perception to memory taking longer. In Experiment 3, the time needed to make a single switch in each direction was examined. The results were not easily described by attention switching: in comparison to within-modality switching, switching from memory to perception took no additional time and switching from perception to memory required additional time only when there was more than one item in memory.

Weber et al. (1986) stated that switching time in a singleswitch situation might not be comparable to switching time in a more continuous task requiring several switches because continuous tasks may require additional capacitydemanding processes. The discrepancy in switching-time estimates between Experiments 1 and 2, which used a continuous task, and Experiment 3, which did not, confirm that suggestion. The data also suggest, though, that the difference results from something other than switching attention between modalities. The question, then, is just what it is that "switching time" reflects.

A similar problem was encountered in an earlier group of studies on switching attention between the two ears in Broadbent's split-span experiments (e.g., Broadbent, 1954; Broadbent \& Gregory, 1961, 1964). Yntema and Trask (1963) accounted for the phenomenon in terms of memory retrieval rather than in terms of attention switching. It is possible to conceptualize the present studies in the same way. The problem is not in accessing the modalities-that is, switching attention-but in selecting the appropriate item once the modality is accessed. Selection is, of course, an attentional phenomenon, but the emphasis is different from the modality-access proposal of Weber et al. (1986).

Consider the data within the working-memory model (Baddeley \& Hitch, 1974) but without the emphasis on moving attention. The system's limited capacity can be used to perform a variety of tasks. To produce an allperception list, the system encodes and immediately outputs the information in the required order. The spatially defined selection of items from the environment is guided by limited-capacity resources. Resources are also required to encode and output the items, but no resources are devoted to storage. To produce an all-memory list, the system encodes the information so that it is in the selected order, and then maintains the items in memory until output. Maintenance in memory requires resources, but the resources necessary for encoding and ordering the items for proper output have been expended before output is required. In Experiments 1 and 2, all-memory lists were produced more quickly than all-perception lists, suggesting that more resources are needed for encoding and selecting than for storing.

To produce mixed lists, the system must expend resources both in maintaining items in memory and in selecting the appropriate items from among those in memory while simultaneously selecting, encoding, and outputting items from the environment. The items in memory are stored in an ordered series. When output is in that order, as in the one-switch lists, selection from memory is just as fast as selection from the environment. With five-switch lists, however, there is a problem. Something such as eye position might indicate the next perception item to be output, but a mechanism is also needed to track information in memory. The data suggest that each time memory is accessed, time is needed to locate the correct item(s) to be output; that is, the to-be-reported item is not immediately available. Yntema and Trask (1963) suggested that the retrieval mechanism has to check some sort of tag for each item to determine whether it has been output. The slower latencies are from retrieval processes occurring after memory access and not from the necessity to switch attention to memory.

Another factor affecting memory retrieval is output interference. As suggested earlier, the perception-first/oneswitch lists were probably more difficult than the memoryfirst/one-switch lists (in both latency and accuracy) because of output interference from reporting the perception items before attempting recall (e.g., see Roediger, 1973, 1974). Output interference would lower accuracy and increase latency in each type of list that requires memory items to be recalled near the end of the list (e.g., Roediger \& Smith, 1980).

The data of Experiment 3 can also be explained in terms of memory retrieval. With the simultaneous cue, the cue is part of the perceptual display and is extremely efficient in guiding the output when a perception item is first. The cue is less efficient for memory-first pairs because it does not directly indicate the appropriate response and must be used as a guide to retrieve items from memory. As might be expected from task demands, items in allmemory lists are stored in pairs so that retrieval of the first memory item allows the second memory item to be retrieved as quickly as a second item can be read off the screen. With perception/memory pairs, in which an item must be selected from among several memory items after a perception item has been output, there is a decrement. 
However, when there is no selection problem because there is only one item in memory, as in the precue group, no attention-switching decrement is found.

Sullivan and Carlson (1989) arrived at a similar conclusion concerning the importance of memory retrieval in a series of experiments examining the individual switching times within continuous switching tasks. Even when their subjects were highly practiced with a particular memory list and could produce it without error, they were slower and less accurate when production of memory items was alternated with production of perception items. The authors suggested that the subjects lost their place in the memory list as a result of having to integrate two lists. In fact, data from an experiment in which subjects were required to integrate information from two perceptual sources suggested to Sullivan and Carlson (Experiment 3) that it is the integration of two lists, and not the switch between modalities, that underlies the difficulty.

Within the Weber et al. (1986) model, production of mixed lists requires the passing of control, or the switching of attention, between memory and perception subroutines. The formulas used to estimate switching time directly reflect the assumption that there is a greater "distance" between a perception item and a memory item than there is between two perception items or two memory items. Weber et al. also suggested, however, that switching time indirectly reflects the extent to which limited resources are needed to implement the switch. The data from the current experiments suggest that it is primarily the indirect processes that are reflected in the switchingtime measure. Within-modality versus between-modality distance between items does not matter in and of itself. Rather, the complexity of the output task in terms of retrieving items from memory appears to be the important factor. The performance decrement found with mixed lists results more from a within-modality selective-attention problem than from between-modality attention switching.

\section{REFERENCES}

BADDELEY, A. (1982). Reading and working inemory. Bulletin of the British Psychological Society, 35, 414-417.
BAdDeley, A. D. , Hitch, G. (1974). Working memory. In G. Bower (Ed.), The psychology of leaming and motivarion (pp. 47-89). London: Academic Press.

BROADBENT, D. E. (1954). The role of auditory localization and attention in memory span. Journal of Experimental Psychology, 47, 191-196.

Broadbent, D. E., Gregory, M. (1961). On the recall of stimuli presented alternately to two sense organs. Quanerly Journal of Experimental Psychology, 13, 103-110.

Broadbent, D. E. Gregory, M. (1964). Stimulus set and response set: The alternation of attention. Quarterly Joumal of Experimental Psychology, 16, 309-317.

ERIKSEN, C. W., St. JAmES, J. D. (1986). Visual attention within and around the field of focal attention: A zoom lens model. Perception \& Psychophysics, 40, 225-240.

Foltz, G. S., Poltrock, S. E. (1985). Apple pascal tester version II (APT II): An experiment development system [Computer program manual]. Austin, TX: Authors.

JONIDES, J. (1981). Voluntary vs. automatic control over the mind's eye's movement. In J. B. Long \& A. D. Baddeley (Eds.), Attention and performance (Vol. 9, pp. 187-203). Hillsdale, NJ: Erlbaum.

Murphy, T. D. ERIKSEN, C. W. (1987). Temporal changes in the distribution of attention in the visual field in response to precues. Perception \& Psychophysics, 42, 576-586.

Poltrock, S. E., Foltz, G. S. (1982). An experimental psychology laboratory system for the Apple II microcomputer. Behavior Research Methods \& Instrumentation, 14, 103-108.

ROEDIGER, H. L., III. (1973). Inhibition in recall from cuing with recall targets. Journal of Verbal Learning \& Verbal Behavior, 12, 644-657.

Roediger, H. L., III. (1974). Inhibiting effects of recall. Memory \& Cognition, 2, 261-269.

Roediger, H. L., III, \& SChmidt, S. R. (1980). Output interference in the recall of categorized and paired-associate lists. Journal of Experimental Psychology: Human Learning \& Memory, 6, 91-105.

Sullivan, M. A. Carlson, R. A. (1989). Attention switching between memory and perceptual information. Unpublished manuscript.

Weber, R. J., BURT, D. B. Noll, N. C. (1986). Attention switching between perception and memory. Memory \& Cognition, 14, 238-245

YNTEMA, D. B., \& Trask, F. P. (1963). Recall as a search process. Journal of Verbal Learning \& Verbal Behavior, 2, 65-74.

\section{NOTE}

1. While recording accuracy, the experimenter also noted trials on which the subject either forgot to hit the space bar or appeared to hit the bar before finishing the list. Those trials were excluded from the analyses as were any response times greater than $6 \mathrm{sec}$. The total amount of data excluded by these criteria was less than $3 \%$ in each experiment.

(Manuscript received December 9, 1988; revision accepted for publication August 10, 1989.) 